\title{
Age at menopause and lung function: a Mendelian randomisation study
}

\author{
Diana A. van der Plaat (10),2,3, Miguel Pereira ${ }^{3}$, Giancarlo Pesce (ib) ${ }^{1,2}$, \\ James F. Potts ${ }^{3}$, André F.S. Amaral $\mathbb{1}^{3}$, Shyamali C. Dharmage $\mathbb{1}^{4}$, \\ Judith M. Garcia-Aymerich (15,6,7, John R. Thompson ${ }^{8}$, Francisco Gómez Real ${ }^{9,10}$, \\ Deborah L. Jarvis $\mathbb{1}^{3}$, Cosetta Minelli $\mathbb{1}^{3,11}$ and Bénédicte Leynaert (1) ${ }^{1,2,11}$ \\ on behalf of the ALEC project
}

\begin{abstract}
Affiliations: 'UMR 1152, Pathophysiology and Epidemiology of Respiratory Diseases, INSERM, Paris, France. ${ }^{2}$ UMR 1152, University Paris Diderot, Paris, France. ${ }^{3}$ National Heart and Lung Institute (NHLI), Imperial College, London, UK. ${ }^{4}$ Allergy and Lung Health Unit, School of Population and Global Health, University of Melbourne, Melbourne, Australia. ${ }^{5}$ SSGlobal, Barcelona, Spain. ${ }^{6}$ Universitat Pompeu Fabra (UPF), Barcelona, Spain. ${ }^{7}$ CIBER Epidemiología y Salud Pública (CIBERESP), Barcelona, Spain. ${ }^{8}$ Dept of Health Sciences, University of Leicester, Leicester, UK. ${ }^{9}$ Dept of Clinical Science, University of Bergen, Bergen, Norway. ${ }^{10}$ Dept of Gynecology and Obstetrics, Haukeland University Hospital, Bergen, Norway. ${ }^{11}$ Contributed equally and are considered to be joint last author.
\end{abstract}

Correspondence: Diana A. van der Plaat, National Heart and Lung Institute (NHLI), Imperial College London, London, UK. E-mail: d.van-der-plaat@imperial.ac.uk

@ERSpublications

Mendelian randomisation, an approach not affected by classical confounding, shows that early menopause has a protective effect on airflow obstruction. This points to the importance of investigating the effects of female sex hormones on the airways. http://bit.ly/2JAZuXh

Cite this article as: van der Plaat DA, Pereira M, Pesce G, et al. Age at menopause and lung function: a Mendelian randomisation study. Eur Respir J 2019; 54: 1802421 [https://doi.org/10.1183/13993003.024212018].

ABSTRACT In observational studies, early menopause is associated with lower forced vital capacity (FVC) and a higher risk of spirometric restriction, but not airflow obstruction. It is, however, unclear if this association is causal. We therefore used a Mendelian randomisation (MR) approach, which is not affected by classical confounding, to assess the effect of age at natural menopause on lung function.

We included 94742 naturally post-menopausal women from the UK Biobank and performed MR analyses on the effect of age at menopause on forced expiratory volume in $1 \mathrm{~s}$ (FEV1), FVC, FEV1/FVC, spirometric restriction (FVC<lower limit of normal (LLN)) and airflow obstruction (FEV1/FVC $<$ LLN). We used the inverse variance-weighted method, as well as methods that adjust for pleiotropy, and compared MR with observational analyses.

The MR analyses showed higher FEV1/FVC and a 15\% lower risk of airflow obstruction for women with early ( $<45$ years) compared to normal (45-55 years) menopause. Despite some evidence of pleiotropy, the results were consistent when using MR methods robust to pleiotropy. Similar results were found among never- and ever-smokers, while the protective effect seemed less strong in women who had ever used menopause hormone treatment and in overweight women. There was no strong evidence of an association with FVC or spirometric restriction. In observational analyses of the same dataset, early menopause was associated with a pronounced reduction in FVC and a 13\% higher risk of spirometric restriction.

Our MR results suggest that early menopause has a protective effect on airflow obstruction. Further studies are warranted to better understand the inconsistency with observational findings, and to investigate the underlying mechanisms and role of female sex hormones.

This article has supplementary material available from erj.ersjournals.com

Received: 20 Dec 2018 | Accepted after revision: 08 July 2019

Copyright OERS 2019 


\section{Introduction}

Women are more susceptible to chronic obstructive pulmonary disease (COPD) than men, especially never-smokers [1], and it is speculated that these sex differences arise partly from distinct sex hormone patterns during life [2]. A major shift in sex hormone levels occurs during menopause. Age at natural menopause shows considerable variability among women, normally occurring between 45 and 55 years of age, with an average of 51 years in European women [3]. As populations age, the proportion of the female population living in a post-menopausal state is increasing and we need to better understand the impact of this major biological event on respiratory health and health in general.

A recent systematic review supports an association of menopause with lower forced expiratory volume in $1 \mathrm{~s}(\mathrm{FEV} 1)$ and lower forced vital capacity (FVC) [4]. A large study in the UK Biobank (UKB) showed that women with an earlier age at menopause had lower FEV1 and FVC, and a higher risk of spirometric restriction, while no association was found with airflow obstruction [5]. These observational studies may suffer from residual confounding even when the analyses are adjusted for known confounders, including anthropometric, lifestyle, socio-economic and reproductive characteristics. Potential confounders may also include early life events, diet and physical activity, and there could be other, yet unrecognised, factors associated with both age at menopause and lung function $[6,7]$.

The Mendelian randomisation (MR) approach can help to assess the causal effect of age at menopause on lung function [8]. With MR, genetic variants, i.e. single nucleotide polymorphisms (SNPs), are used as proxies ("instrumental variables") for age at menopause [9]. The alleles of a genetic variant are randomly allocated at conception and therefore not affected by classical confounding. Finding that the SNPs predisposing to earlier age at menopause are associated with lung function would provide indirect evidence of a causal effect, as long as the assumptions underlying MR hold (figure 1) [8]. Of particular concern is pleiotropy, which is present when an SNP is also associated with other traits affecting lung function, independently from age at menopause $[8,10]$.

In the current study, we used MR to estimate the effect of age at menopause on five lung function outcomes (FVC, FEV1, FEV1/FVC, spirometric restriction and airflow obstruction) using data from the $\mathrm{UKB}$. We categorised menopause as early, normal and late to allow for the investigation of possible nonlinear effects. Early menopause is of particular clinical interest and has been most commonly investigated for associations with health outcomes in previous studies. We assessed the robustness of our main MR results using methods to investigate and adjust for pleiotropy, and we performed subgroup analyses to investigate whether the effect of age at menopause on lung function varied according to smoking status, use of menopause hormone therapy (MHT) and body mass index (BMI). We also performed classical observational analyses on the same dataset, for comparison with MR.

\section{Methods}

Detailed methods are provided in the supplementary data.

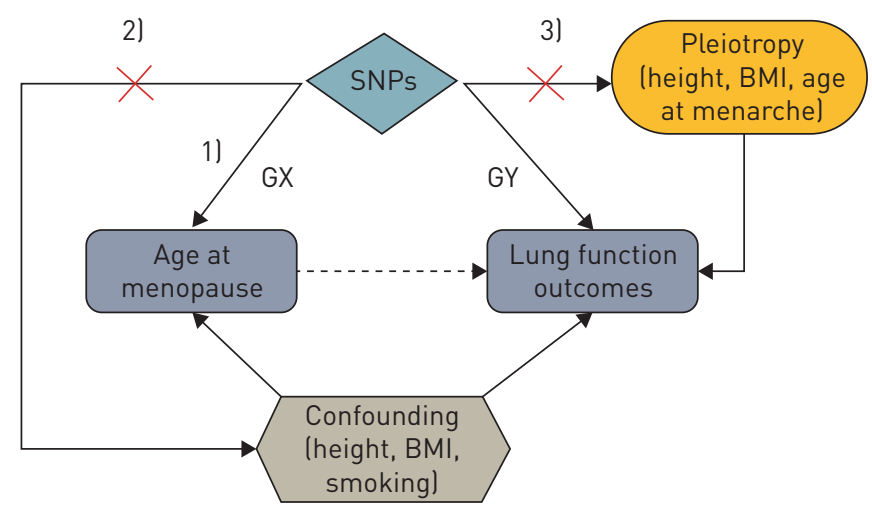

FIGURE 1 Schematic overview of Mendelian randomisation (MR). For each single nucleotide polymorphism (SNP), the MR effect estimate is calculated using the GX lassociation of the SNP with early and late menopause versus normal menopause) and GY lassociation of the SNP with lung function outcomes) associations. MR assumptions: 1) relevance assumption: SNPs are associated with exposure lage at menopause); 2) independent assumption: the association between SNP and outcome is NOT confounded; and 3) exclusion restriction assumption: SNPs affect outcome (lung function) only through the exposure and not through independent pathways (i.e. absence of pleiotropy). BMI: body mass index. 


\section{Study population}

We included naturally post-menopausal white women from the very large, population-based UKB study [11], and excluded women with surgical menopause and women with extreme age at menopause ( $<40$ years or $>60$ years). Age at natural menopause was based on self-reported age at which "periods stopped", and was categorised as early (40-45 years), normal (45-55 years), or late (55-60 years), using normal as the reference category. We investigated the continuous outcomes FEV1, FVC and FEV1/FVC (best measure), as well as the binary outcomes of spirometric restriction defined as FVC<lower limit of normal (LLN) and airflow obstruction defined as FEV1/FVC $<$ LLN. The LLN was based on the Global Lung Function Initiative 2012 equations [12].

\section{SNP selection}

Based on published genome-wide association studies, we identified 80 SNPs associated with continuous age at menopause at a p-value threshold of $5 \times 10^{-8}$ [13-18]. After removing SNPs with F-statistic $\leqslant 10$ to avoid weak instrument bias and SNPs highly correlated with each other $\left(r^{2} \geqslant 0.8\right), 63$ SNPs remained that jointly explained $6 \%$ of the UKB population variance of age at menopause [19]. Of the selected SNPs, 39 SNPs were strong instruments for early menopause and 40 for late menopause.

\section{MR analyses}

As our main analysis, we estimated the causal effect of early and late menopause on lung function. As secondary analyses, we performed MR analyses considering age at menopause as a continuous variable. For each of the included SNPs we obtained two estimates from UKB (figure 1): one for the association of the SNP with early and late menopause versus normal menopause (GX), using logistic regression adjusted for centre, first 10 ancestry principal components and three genotyping batches; and one for the association with the lung function outcomes (GY), using logistic (airway obstruction and spirometric restriction) or linear (FEV1/FVC, FVC and FEV1) regression adjusted for age, age ${ }^{2}$, height, centre, 10 principal components and three batches. For each SNP, an MR estimate was derived as the ratio of GY over GX (Wald estimator) and its standard error obtained using the Delta method [20]. SNP-specific MR estimates were then pooled using an inverse variance-weighted (IVW) fixed-effect meta-analysis method that accounts for correlation between SNPs ( $\mathrm{R}$ package MendelianRandomization; R Foundation for Statistical Computing, Vienna, Austria) [19, 21]. We used the IVW two-sample MR method because the standard MR approach in a one-sample setting (as in our study), which is the two-stage least squares (2SLS) method, does not account for pleiotropy.

\section{Assessing pleiotropy}

The presence of pleiotropy was investigated using between-instrument heterogeneity of the IVW MR estimates, based on the $\mathrm{I}^{2}$ statistic and Q test [22]. In secondary analyses we investigated the robustness of our findings to pleiotropy using MR methods based on different assumptions about the direction of possible pleiotropic effects: 1) IVW random-effect meta-analysis (assumes random pleiotropic effects); 2) weighted median analysis (assumes that at least 50\% of the SNPs are valid inverse variances with no assumption on the direction of pleiotropic effects); 3) MR-Egger regression with penalised weights (assumes directional pleiotropic effects); and 4) MR-PRESSO (corrects for horizontal pleiotropy by removing pleiotropic outlier SNPs) [10, 23-25]. Furthermore, we a priori considered height, BMI and age at menarche as potential sources of pleiotropy. We excluded SNPs that were associated with these traits at a p-value $<7.9 \times 10^{-4}$ (Bonferroni correction, 0.05/63) based on information from the online PhenoScanner [26].

\section{Sensitivity analyses}

We performed several sensitivity analyses to assess the robustness of our findings to choices made $a$ priori for our MR analyses. These related to the MR method used, the sample used and the choice of SNPs used as instruments: 1) we performed the standard MR analysis for the one-sample setting, i.e. the 2SLS regression analysis, which assumes no pleiotropy similarly to the IVW fixed-effect method [27]; 2) we restricted the main MR analysis to exactly the same number of women included in the observational analysis to allow comparison between the two approaches $(n=84844) ; 3)$ we excluded asthmatic women because these women may have reversible airflow obstruction; and 4) we limited the MR analyses to the 54 SNPs identified by the most recent and largest genome-wide association study on age at menopause [18] and did not adjust for correlation (the 54 SNPs are independent) or remove weak SNPs in these analyses. Finally, we used GX estimates for continuous age at menopause from DAY et al. [18], together with the GY estimates from $\mathrm{UKB}$, to further investigate the robustness of our findings when using two independent data sources (two-sample MR) instead of a single dataset (one-sample MR). 


\section{Subgroup analyses}

For the lung function outcomes with consistently significant findings, we performed subgroup analyses by smoking status (never versus ever) because some evidence suggests that a longer exposure to female sex hormones might increase airway susceptibility to the effects of smoking [28, 29]. In addition, we investigated potential effect modification by MHT use (never versus ever) and BMI (low versus high based on a cut-off of $26 \mathrm{~kg} \cdot \mathrm{m}^{-2}$ (median)), because women taking hormonal therapy and obese women may still have high levels of female sex hormones after menopause.

\section{Observational analyses}

Earlier observational analyses in UKB examined age at menopause as a categorical variable divided into five groups [5]. For comparison with our MR findings, we performed observational analyses in UKB using three menopause categories. Moreover, in addition to confounders previously considered (centre, age, age ${ }^{2}$, height, BMI, smoking groups, Townsend deprivation index, MHT use, number of live births, ever use of oral contraceptives and age at menarche), we also included physical activity and education level $[6,7,30]$.

All analyses were performed using R (R Foundation for Statistical Computing).

\section{Results}

Population characteristics

From the UKB, 94742 white women who had undergone natural menopausal were included in the MR analyses, of which 7206 had early and 8468 had late menopause (table 1 and supplementary table S1). The mean age was 60 years and mean age at menopause 51 years; $57 \%$ of women had never used MHT, $7 \%$ had spirometric restriction (FVC $<$ LLN) and $8 \%$ had airflow obstruction $(\mathrm{FEV} / / \mathrm{FVC}<\mathrm{LLN})$. Women with late menopause were less likely to have ever smoked or to have ever used MHT compared to women with early menopause. Only 84844 of these women had complete data on all confounders and were included in the observational analyses, and their characteristics were the same as those of the MR sample (table 1 and supplementary table S1).

TABLE 1 Characteristics of the study population

\begin{tabular}{|c|c|c|c|c|}
\hline & $\begin{array}{l}\text { Women included } \\
\text { in MR }\end{array}$ & $\begin{array}{l}\text { Women with } \\
\text { complete data\# }\end{array}$ & $\begin{array}{l}\text { Women with early } \\
\text { menopause }\end{array}$ & $\begin{array}{c}\text { Women with late } \\
\text { menopause }\end{array}$ \\
\hline Subjects $\mathrm{n}$ & 94742 & 84844 & 7206 & 8468 \\
\hline Age years & $60 \pm 5.3$ & $60 \pm 5.3$ & $59 \pm 6.8$ & $62 \pm 3.4$ \\
\hline Height cm & $162 \pm 6.1$ & $162 \pm 6.1$ & $162 \pm 6.2$ & $162 \pm 6.0$ \\
\hline $\mathrm{BMI} \mathrm{kg} \cdot \mathrm{m}^{-2}$ & $26.9 \pm 4.9$ & $26.8 \pm 4.8$ & $27.0 \pm 5.0$ & $27.5 \pm 5.0$ \\
\hline Age at menopause years & $51 \pm 4.0$ & $51 \pm 4.0$ & $42 \pm 1.4$ & $57 \pm 1.2$ \\
\hline Early menopause $(<45$ years $)$ & $7206(7.6)$ & $6369(7.5)$ & $7206(100)$ & \\
\hline Late menopause (>55 years) & $8468(8.9)$ & 7541 (8.9) & & $8468(100)$ \\
\hline Age at menarche years & $13 \pm 1.6$ & $13 \pm 1.6$ & $13 \pm 2.9$ & $13 \pm 2.4$ \\
\hline \multicolumn{5}{|l|}{ Lung function } \\
\hline FVC \% pred & $97.4 \pm 15.7$ & $97.7 \pm 15.6$ & $95.7 \pm 15.9$ & $97.5 \pm 15.6$ \\
\hline FEV $1 \%$ pred & $93.1 \pm 16.8$ & $93.4 \pm 16.7$ & $91.1 \pm 17.3$ & $93.3 \pm 16.8$ \\
\hline $\mathrm{FEV}_{1} / \mathrm{FVC}$ & $75.3 \pm 6.3$ & $75.3 \pm 6.3$ & $75.1 \pm 6.7$ & $75.2 \pm 6.3$ \\
\hline $\mathrm{FEV}_{1} / \mathrm{FVC}<\mathrm{LLN}$ & $7979(8.4)$ & 7010 (8.3) & $727(10)$ & $637(7.5)$ \\
\hline$F V C<L L N$ & $6869(7.3)$ & $5892(6.9)$ & $650(9.0)$ & $585(6.9)$ \\
\hline Asthma? & $10900(12)$ & $9726(11)$ & $926(13)$ & $993(12)$ \\
\hline \multicolumn{5}{|l|}{ Smoking status } \\
\hline Never-smoker & $53831(57)$ & $48393(57)$ & 3633 (50) & 4995 (59) \\
\hline Ex-smoker & $33518(35)$ & $30194(36)$ & 2685 (37) & $3016(36)$ \\
\hline Current-smoker & $7370(8)$ & $6257(7)$ & $884(12)$ & $455(5)$ \\
\hline Pack-years in smokers & $21 \pm 16$ & $20 \pm 15$ & $24 \pm 16$ & $20 \pm 16$ \\
\hline \multicolumn{5}{|l|}{ MHT } \\
\hline Never used & 53946 (57) & $48395(57)$ & $3201(45)$ & $4390(52)$ \\
\hline Ex-user & 36261 (38) & 32407 (38) & 3459 (48) & $3739(44)$ \\
\hline Current user & 4375 (5) & $4042(5)$ & $532(7)$ & $328(4)$ \\
\hline
\end{tabular}

Data are reported as mean \pm SD or $\mathrm{n}(\%)$, unless otherwise stated. See supplementary table $\mathrm{S} 1$ for more variables and subgroups. MR: Mendelian randomisation; BMI: body mass index; FVC: forced vital capacity; FEV1: forced expiratory volume in $1 \mathrm{~s}$; LLN: lower limit of normal; MHT: menopause hormone therapy. \#: women with complete data on all variables included in the observational analyses; ": self-reported doctor-diagnosed asthma ever. 
TABLE 2 Association of early menopause with airflow obstruction in observational and Mendelian randomisation (MR) analyses

\begin{tabular}{|c|c|c|c|c|c|c|c|c|}
\hline & \multirow[t]{2}{*}{ Subjects $\mathrm{n}$} & \multirow[t]{2}{*}{ SNPs $\mathrm{n}$} & \multicolumn{3}{|c|}{ FEV1/FVC \% } & \multicolumn{3}{|c|}{ FEV $1 / F V C<L L N$} \\
\hline & & & B $(95 \% \mathrm{CI})$ & p-value & $\mathrm{I}^{2}(95 \% \mathrm{Cl})$ & OR $(95 \% \mathrm{CI})$ & p-value & $\mathrm{I}^{2}(95 \% \mathrm{Cl})$ \\
\hline Observational analysis & 77303 & & $\begin{array}{c}-0.19 \\
(-0.34--0.03)\end{array}$ & 0.019 & NA & $1.09(1.00-1.19)$ & 0.063 & NA \\
\hline Main MR analysis (all SNPs) & 86274 & 39 & $0.29(0.22-0.36)$ & $1.48 \times 10^{-16}$ & $48(34-83)$ & $0.85(0.82-0.89)$ & $5.88 \times 10^{-14}$ & $16(0-60)$ \\
\hline \multicolumn{9}{|l|}{ Pleiotropic SNPs excluded } \\
\hline Height & 86274 & 30 & $0.28(0.21-0.36)$ & $2.29 \times 10^{-13}$ & $56(47-88)$ & $0.84(0.81-0.88)$ & $1.44 \times 10^{-13}$ & $26(0-70)$ \\
\hline All $^{\#}$ & 86274 & 28 & $0.29(0.22-0.37)$ & $7.13 \times 10^{-14}$ & 57 (49-89) & $0.84(0.80-0.88)$ & $1.19 \times 10^{-13}$ & $30(0-74)$ \\
\hline \multicolumn{9}{|l|}{ Sensitivity MR analyses } \\
\hline Women with complete data" & 77303 & 39 & $0.28(0.21-0.35)$ & $1.86 \times 10^{-14}$ & $45(25-80)$ & $0.87(0.84-0.91)$ & $7.14 \times 10^{-10}$ & $14(0-58)$ \\
\hline Non-asthmatic women & 76367 & 39 & $0.31(0.24-0.38)$ & $7.47 \times 10^{-18}$ & $42(19-80)$ & $0.84(0.80-0.88)$ & $2.07 \times 10^{-12}$ & $7(0-48)$ \\
\hline $\begin{array}{l}\text { Limiting to Day et al. [18] } \\
\text { SNPs }\end{array}$ & 86274 & 33 & $0.29(0.18-0.39)$ & $3.75 \times 10^{-8}$ & $38(5-77)$ & $0.89(0.84-0.95)$ & $2.20 \times 10^{-4}$ & $4(0-54)$ \\
\hline
\end{tabular}

Significant results are shown in bold. FEV1: forced expiratory volume in $1 \mathrm{~s}$; FVC: forced vital capacity; LLN: lower limit of normal; SNP: single nucleotide polymorphism; $I^{2}$ : between-instrument heterogeneity indicator; BMI: body mass index; NA: not applicable. \#: all pleiotropic SNPs associated with height, BMI and age at menarche excluded from the analysis and SNPs associated with the traits were selected based on the results from the PhenoScanner with $p<7.94 \times 10^{-4}(0.05 / 63)$; ": women with complete data on all variables included in the observational analyses.

Detailed results of all MR and observational analyses, separately for early and late menopause, can be found in supplementary table S2 (GX and GY effect estimates) and supplementary table S3 (MR results).

\section{MR analyses}

For early menopause (table 2), the MR analyses showed higher FEV1 (5.55 mL, 95\% CI 0.92-10.18 mL), higher FEV1/FVC $(0.29 \%$, 95\% CI $0.22 \%-0.36 \%)$ and a $15 \%$ lower risk of airflow obstruction (OR 0.85 , 95\% CI 0.82-0.89), as compared to normal menopause (figure 2 and supplementary table S3). No effect of early menopause was found for FVC $(-4.98 \mathrm{~mL}, 95 \% \mathrm{CI}-10.5-0.53 \mathrm{~mL})$ or spirometric restriction (OR $1.03,95 \%$ CI $0.99-1.08)$.

For late menopause (table 3), the MR analyses showed lower FEV1 $(-11.0 \mathrm{~mL}, 95 \% \mathrm{CI}-16.4--5.68 \mathrm{~mL})$ and lower $\mathrm{FEV} / \mathrm{FVC}(-0.18 \%, 95 \% \mathrm{CI}-0.26 \%--0.10 \%)$, and moderately higher risk of airflow obstruction (OR 1.06, 95\% CI 1.01-1.11) (figure 2 and supplementary table S3). Late menopause was also borderline significantly associated with lower FVC $(-7.10 \mathrm{~mL}, 95 \% \mathrm{CI}-13.5--0.75 \mathrm{~mL})$ but not with spirometric restriction (OR 1.00, 95\% CI 0.95-1.05).

These results were consistent when considering age at menopause as a continuous variable, with an increase in age at menopause associated with lower $\mathrm{FEV}$, lower $\mathrm{FEV}$ //FVC and a higher risk of obstruction (supplementary table S4).

\section{Assessing pleiotropy}

Heterogeneity was low for the analyses on spirometric restriction and airflow obstruction $\left(\mathrm{I}^{2}=0 \%-20 \%\right.$; $\mathrm{Q} p=0.098-3.2 \times 10^{-4}$; global test $\left.\mathrm{p}=0.05-0.22\right)$. For the continuous lung function outcomes, the high $\mathrm{I}^{2}$ $(46 \%-53 \%)$, the significant $Q$ test $\left(p=1.0 \times 10^{-5}-5.2 \times 10^{-10}\right)$ and significant global test of MR-PRESSO $(\mathrm{p}<0.0005)$ suggested the presence of (horizontal) pleiotropy (supplementary table S3). Correcting for horizontal pleiotropy by MR-PRESSO increased the effect estimate sizes but these were not significantly different from the original MR estimates (distortion test $\mathrm{p}=0.43-0.94$ ).

Overall, the results from the other MR methods robust to pleiotropy were consistent with those from the main analysis (figure 2 and supplementary table S3). For FEV1/FVC and airflow obstruction, a protective effect of early menopause was found for all MR methods. For FVC and spirometric restriction, the MR estimates remained nonsignificant, except for the MR-Egger estimate of early menopause on spirometric restriction (OR 1.12, 95\% CI 1.00-1.24).

We further assessed the impact of pleiotropy by removing SNPs with potential pleiotropic effects. Out of 63 SNPs, 16 SNPs have previously been associated with height, five with (adult) BMI and four with age at menarche. Removing these SNPs gave similar results for both FEV1/FVC and airflow obstruction compared to the main analyses, indicating robustness to pleiotropy (tables 2 and 3 and supplementary table S3). However, we found a 

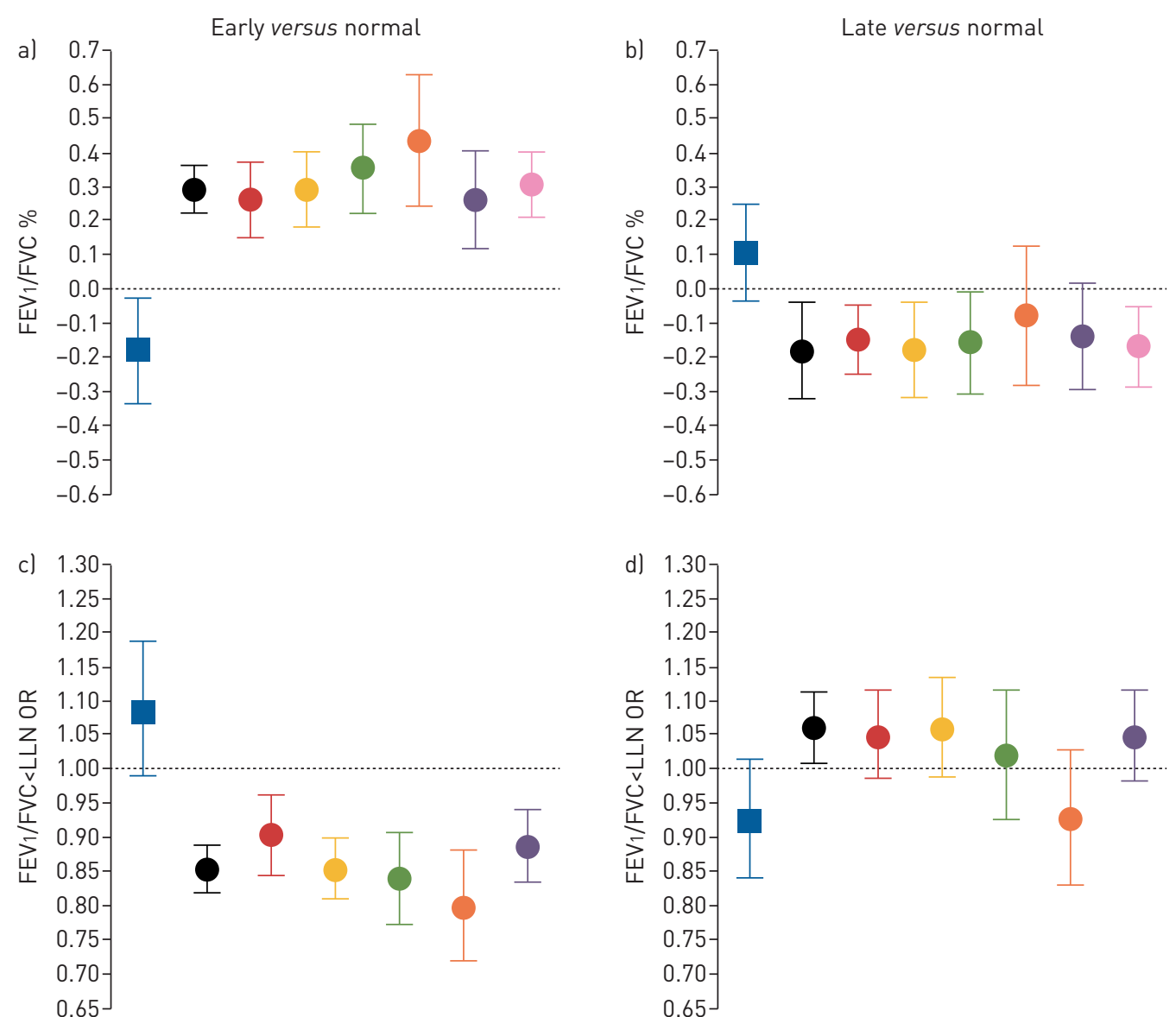

0.65

Observational analysis

MR-IVW random effect

MR-PRESSO (raw)
MR-IVW fixed effect (main)

MR-Weighted median

MR-PRESSO (corrected)

FIGURE 2 Association of early and late menopause with airflow obstruction in observational and Mendelian randomisation (MR) analyses. a, c) Early menopause: forced expiratory volume in $1 \mathrm{~s}(\mathrm{FEV} 1) /$ forced vital capacity (FVC) (a) and airflow obstruction as measured by $F E V_{1} / F V C<$ lower limit of normal (LLN) (c). b, d) Late menopause: $F E V_{1} / F V C(b)$ and $F E V_{1} / F V C<L L N(d)$. The observational analyses (linear/logistic regression) were adjusted for age, age ${ }^{2}$, height, centre, body mass index, smoking groups, Townsend deprivation index, level of education, physical activity, number of live births, age at menarche, ever oral contraceptive use and use of menopause hormone therapy. All MR analyses were adjusted for age, age ${ }^{2}$, height, centre, first 10 principal components and genotyping batch. Data are presented with $95 \% \mathrm{Cl}$. For MR-PRESSO, both unadjusted analyses (raw) and analyses after removal of outliers if any were detected (corrected) are shown. IVW: inverse variance-weighted average method; 2SLS: two-stage least squares regression.

small detrimental effect of early menopause on FVC when excluding possible pleiotropic SNPs $(-6.77 \mathrm{~mL}$, $95 \% \mathrm{CI}-12.8--0.067 \mathrm{~mL}$ ), but still no effect on spirometric restriction (OR 1.03, 95\% CI 0.98-1.08).

\section{Sensitivity analyses}

Results consistent with the main analyses were found in all sensitivity analyses assessing the robustness of our findings to choices made a priori (tables 2 and 3 for FEV1/FVC and airflow obstruction; supplementary table S3 for all outcomes). These related to the MR method used (one-sample 2SLS (figure $2)$ ), the sample used (same number as observational analyses and limited to non-asthmatic women), and the choice of SNPs used as instruments (limiting to SNPs identified by DAY et al. [18]). We also found consistent results when using GX estimates for continuous age at menopause from the literature [18] and GY estimates from UKB (supplementary table S4).

\section{Subgroup analyses}

We hypothesised a possible stronger effect in smokers, but found no statistically significant difference in MR estimates for FEV1/FVC and airflow obstruction between ever- and never-smokers (figure 3 and supplementary table S5). In addition, we hypothesised that the protective effect of early menopause might 
TABLE 3 Association of late menopause with airflow obstruction in observational and Mendelian randomisation (MR) analyses

\begin{tabular}{|c|c|c|c|c|c|c|c|c|}
\hline & \multirow[t]{2}{*}{ Subjects $\mathrm{n}$} & \multirow[t]{2}{*}{ SNPs $\mathrm{n}$} & \multicolumn{3}{|c|}{ FEV $1 /$ FVC \% } & \multicolumn{3}{|c|}{$\mathrm{FEV}_{1} / \mathrm{FVC}<\mathrm{LLN}$} \\
\hline & & & $\beta(95 \% \mathrm{Cl})$ & p-value & $\mathrm{I}^{2}(95 \% \mathrm{CI})$ & OR $(95 \% \mathrm{CI})$ & p-value & $I^{2}(95 \% \mathrm{CI})$ \\
\hline Observational analysis & 78475 & & $0.11(-0.04-0.25)$ & 0.139 & NA & $0.92(0.84-1.02)$ & 0.101 & NA \\
\hline \multicolumn{9}{|l|}{ Pleiotropic SNPs excluded } \\
\hline Height & 87536 & 31 & $-0.22(-0.33--0.11)$ & $6.90 \times 10^{-5}$ & $61(49-86)$ & $1.10(1.03-1.17)$ & $4.53 \times 10^{-3}$ & $23(0-60)$ \\
\hline $\mathrm{BMI}$ & 87536 & 37 & $-0.17(-0.26--0.09)$ & $3.13 \times 10^{-5}$ & $54(44-85)$ & $1.06(1.01-1.11)$ & 0.027 & $20(0-59)$ \\
\hline \multicolumn{9}{|l|}{ Sensitivity MR analyses } \\
\hline Women with complete data? & 78475 & 40 & $-0.17(-0.25--0.08)$ & $9.63 \times 10^{-5}$ & $53(44-84)$ & $1.05(1.00-1.11)$ & 0.042 & $13(0-51)$ \\
\hline Non-asthmatic women & 77562 & 40 & $-0.18(-0.26--0.10)$ & $1.79 \times 10^{-5}$ & $53(44-83)$ & $1.06(1.00-1.12)$ & 0.044 & $6(0-48)$ \\
\hline Limiting to DAY et al. [18] & 87536 & 33 & $-0.17(-0.27--0.08)$ & $4.39 \times 10^{-4}$ & $44(20-83)$ & $1.05(0.99-1.12)$ & 0.077 & $6(0-52)$ \\
\hline SNPs & & & & & & & & \\
\hline
\end{tabular}

Significant results are shown in bold. FEV1: forced expiratory volume in $1 \mathrm{~s}$; FVC: forced vital capacity; LLN: lower limit of normal; SNP: single nucleotide polymorphism; $I^{2}$ : between-instrument heterogeneity indicator; BMI: body mass index; NA: not applicable. \#: all pleiotropic SNPs associated with height, $\mathrm{BMI}$ and age at menarche excluded from the analysis and SNPs associated with the traits were selected based on the results from the PhenoScanner with $p<7.94 \times 10^{-4}(0.05 / 63)$; ": women with complete data on all variables included in the observational analyses.

be less evident in women who still had high levels of female sex hormones after menopause (i.e. MHT users and overweight/obese women), and our findings support this hypothesis (figure 3 and supplementary table S5). We found a significant difference in effect between never- and ever-MHT users; the interaction was significant for airflow obstruction for both early and late menopause ( $\mathrm{p}=0.002$ and $0.004)$, while for $\mathrm{FEV} 1 / \mathrm{FVC}$ the interaction was significant for early menopause $(\mathrm{p}=0.04)$ and borderline for late menopause $(p=0.07)$. In the subgroup analyses based on median BMI, the interaction was significant for FEV1/FVC for both early and late menopause $\left(p=4.6 \times 10^{-7}\right.$ and 0.005$)$. For airflow obstruction, the interaction was significant for early menopause $(p=0.026)$ and borderline for late menopause $(\mathrm{p}=0.07)$.

\section{Observational analyses}

In the observational analyses, women who experienced early menopause showed statistically significantly lower FEV1 $(-31.4 \mathrm{~mL}$, 95\% CI-42.0- -20.9 mL), lower FVC $(-36.3 \mathrm{~mL}, 95 \% \mathrm{CI}-48.8--23.8 \mathrm{~mL})$ and a higher risk of spirometric restriction (OR 1.13, 95\% CI 1.02-1.24) (figure 2 and supplementary table S3). Early menopause was also associated with lower FEV1/FVC, although the confidence interval was very large $(-0.19 \%, 95 \%$ CI $-0.34 \%--0.03 \%)$, and possibly a higher risk of airflow obstruction (OR $1.09,95 \%$
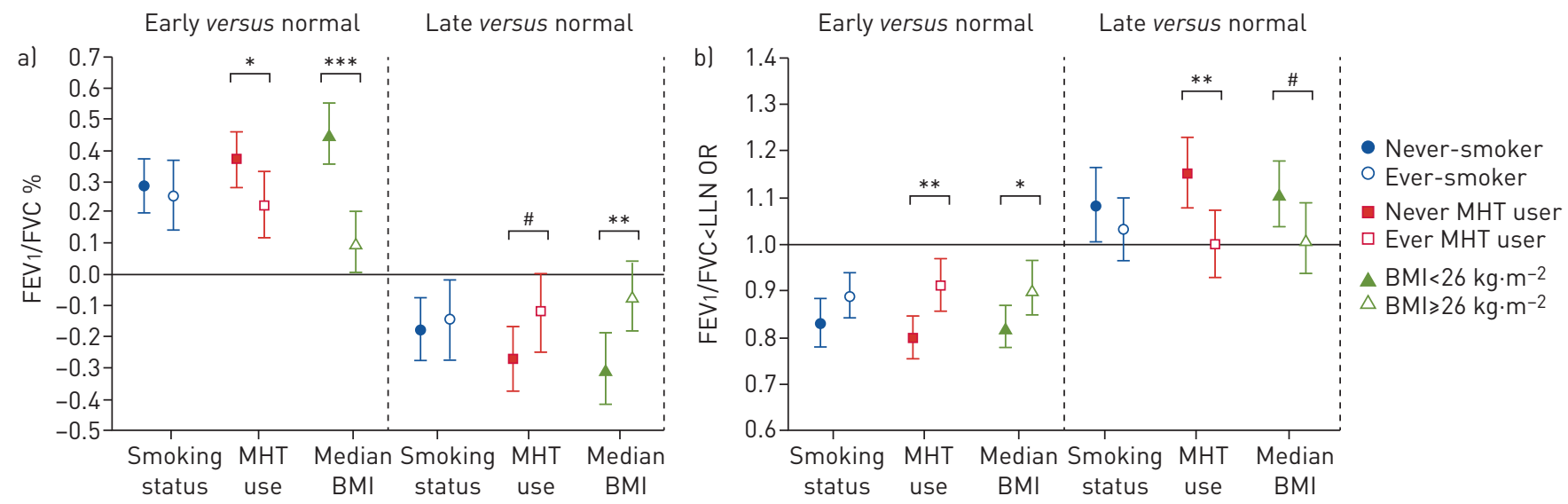

FIGURE 3 Mendelian randomisation estimates of the effect of age at menopause on a) forced expiratory volume in $1 \mathrm{~s}$ (FEV 1 )/forced vital capacity (FVC) and b) airflow obstruction as measured by $\mathrm{FEV}_{1} / \mathrm{FVC}<$ lower limit of normal (LLN) in subgroup analyses by smoking status, menopause hormone therapy (MHT) use and current body mass index (BMI). Data are presented with $95 \% \mathrm{Cl} .{ }^{\#}: p<0.10 ;{ }^{*}: p<0.05 ;{ }^{* *}$ : $p<0.01 ;{ }^{* * *}$ : $p<0.001$. 
CI 1.00-1.19) that did not reach statistical significance. Late menopause was not associated with any of the five lung function outcomes.

\section{Discussion}

Contrary to observational findings, our MR study suggests that early menopause has a protective effect on airflow obstruction, with no clear evidence of an effect on FVC or spirometric restriction [5, 31-35]. We found a $15 \%$ decrease in the risk of airflow obstruction for women with menopause before the age of 45 years compared to women with a normal age at menopause, and this protective effect was less strong in women who had used MHT as well as in overweight women. Although there was some evidence of pleiotropy, overall our findings were consistent when using MR methods robust to pleiotropy.

MR studies can be considered similar to randomised controlled trials (RCTs) in that exposure to a specific genetic variant is assigned randomly at conception. MR estimates are therefore not affected by classical confounding factors. However, MR studies can provide evidence of a causal effect only if the three key assumptions underlying MR hold (figure 1) [36, 37]. The first assumption (relevance assumption) is that the SNPs used as instruments are associated with menopause, and we strengthened this by removing weak instruments (F-statistic <10). The second assumption (independence assumption) is that the association between the SNP and the outcome is not confounded. The third, and most important, assumption (exclusion restriction) is the absence of pleiotropy, i.e. the SNP is not associated with other traits that independently affect the outcome. Related to assumption 2, the main confounder for genetic associations is population stratification, and we addressed this by adjusting for ancestry principal components. The impact of violations of assumptions 2 and 3 can be assessed by excluding SNPs known to be associated with traits that could affect lung function. We a priori considered height, BMI and age at menarche as potential sources of confounding and/or pleiotropy because these traits are associated with lung function, and $25 \%, 8 \%$ and $6 \%$ respectively of the 63 included SNPs have been previously associated with these traits. Removing these SNPs resulted in similar results to those of the main analyses, suggesting no major impact on the MR results through genetic associations of these traits. The presence of pleiotropy can also be investigated by looking at the variation between MR estimates from the individual SNPs. We would expect the individual MR estimates to vary only by chance if all included SNPs were valid instruments (i.e. no pleiotropy) [22]. There was a suggestion of pleiotropy in our main analyses on FEV1/FVC, FVC and airflow obstruction $\left(\mathrm{I}^{2}=16-48\right)$, but not for spirometric restriction $\left(\mathrm{I}^{2}=0\right)$. However, when we excluded possible pleiotropic SNPs, there was still residual heterogeneity $\left(\mathrm{I}^{2}=2-57\right)$, which could be due to other (unknown) independent pathways. It is important to note that $\mathrm{I}^{2}$ is not independent of sample size or number of instruments, and with large sample sizes high $\mathrm{I}^{2}$ values might be observed in the absence of pleiotropy [22]. The consistency between the findings of the different MR methods provides reassurance and indicates the robustness of our findings related to airflow obstruction.

Our findings when using age at menopause as a continuous variable were consistent with those for early and late menopause. Of note, this MR analysis had 90\% power to detect a minimum difference in FVC of $6.4 \mathrm{~mL}$ per year increase in age at menopause, and hence we cannot exclude a smaller effect of age at menopause on FVC [38]. Our main analyses suggested the possibility of a small detrimental effect of early menopause on FVC $(-5 \mathrm{~mL}, \mathrm{p}=0.08)$. When we excluded possible pleiotropic SNPs, this association became significant $(\mathrm{p}=0.03)$ and its direction was the same as in the observational analysis, although of much smaller magnitude $(-6.7 \mathrm{~mL}$ versus $-36 \mathrm{~mL})$. We thus cannot confirm the detrimental effect of early menopause on FVC found in observational analyses, but if there is a true causal effect this is likely to be small.

Most observational studies report lower FEV1 and FVC in post-menopausal women compared to pre-menopausal, but no change in FEV1/FVC or airflow obstruction [5, 31-35]. AmARAL et al. [5] studied the effect of age at menopause (categorised into five groups) on lung function in the UKB and found that an earlier age at menopause associated with lower FVC and FEV1, and with a higher risk of spirometric restriction. In line with this study, but focusing on early and late menopause and adjusting for additional potential confounders, our observational analysis in UKB showed strong associations of early menopause with lower FVC and a 13\% higher risk of spirometric restriction. Based on the same UKB study population but using MR, we found strong associations of early menopause with higher FEV1/FVC and a $15 \%$ lower risk of airflow obstruction. We could not confirm a strong effect on FVC nor spirometric restriction using MR. We may have found a protective effect of early menopause on airflow obstruction in MR that we did not find in observational studies because of confounding of the observational findings by factors that increase the risk of early menopause and decrease lung function, which could include early life factors, diet and occupational exposures. Confounding might also explain why early menopause is associated with a stronger reduction in FVC in observational analyses compared to MR. Future studies that can account for more potential confounders could help explain the inconsistency between observational and MR findings. 
Menopause marks the end of the reproductive period, which is defined as the time between menarche and menopause. Age at menopause was highly correlated with the length of the reproductive period in our data (correlation coefficient of 0.93). Early menopause might thus reflect the effect of a shorter lifetime "exposure" to female sex hormones. We would expect less of an effect of early menopause on airflow obstruction in women who still have high levels of female sex hormones after menopause, including women taking hormonal therapies and obese women with higher endogenous levels. We therefore performed subgroup analyses by MHT use and by BMI, and found that the effect of early menopause on airflow obstruction was not so apparent in these groups, supporting this hypothesis. The role of sex hormones in the lung is complex and there are biological reasons to support either a beneficial or a detrimental effect depending on the type of hormone, concentration, duration of exposure, cell type and their interactions $[2,39]$. Beneficial effects of hormone therapy on lung function after 3-6 months MHT use have been reported by small RCTs, while a crossover RCT reported no improvement in lung function after MHT use [40-43]. Previous research in mice has shown that oestrogens may potentiate the oxidative stress after smoking exposure, and thereby contribute to airway remodelling [29]. We therefore performed subgroup analyses by smoking status, but found no evidence of a difference between smokers and non-smokers.

Although technically the reproductive period is also defined by menarche, age at menarche varies very little compared to age at menopause and therefore influences lifetime exposure to sex hormones to a much lesser extent than age at menopause. Interestingly, an earlier age at menarche has previously been associated with a reduced FVC in adult women in a previous MR study, while no association was found for FEV1/FVC [38]. The proposed mechanism for the effect of early menarche relates to premature completion of lung development and a lower maximal attained lung function, which has been confirmed by an observational longitudinal study [44]. Therefore, the effect of age at menarche on lung function might not be through sex hormones, unlike what we hypothesised for age at menopause.

A limitation of our study is the use of self-reported information on age at menopause. However, the misclassification is likely to be random and the measurement error would reduce power rather than causing bias. UKB did not perform post-bronchodilator pulmonary function measurements, so we cannot distinguish between reversible and fixed airflow obstruction. When we excluded patients with self-reported asthma from the MR analyses, we found almost identical results compared to the main analysis. Strengths of our study are the large sample size, which is needed for MR, and the $6 \%$ variance in age at menopause explained by the SNPs used in our MR, which is relatively high.

In conclusion, this MR study suggests that the earlier a woman's menopause, the less likely she is to have airflow obstruction. Future research is needed to further assess the effect of female sex hormones and the length of the reproductive period on lung function. In addition, our MR study could not confirm a strong effect of early menopause on FVC and spirometric restriction as found in observational studies, and further work might explain this inconsistency.

Conflict of interest: D.A. van der Plaat has nothing to disclose. M. Pereira has nothing to disclose. G. Pesce has nothing to disclose. J.F. Potts has nothing to disclose. A.F.S. Amaral has nothing to disclose. S.C. Dharmage has nothing to disclose. J.M. Garcia-Aymerich has nothing to disclose. J.R. Thompson has nothing to disclose. F. Gómez Real has nothing to disclose. D.L. Jarvis reports grants from European Union, during the conduct of the study. C. Minelli has nothing to disclose. B. Leynaert has nothing to disclose.

Support statement: The current study is part of the Ageing for Lungs in European Cohorts (ALEC) study (www. alecstudy.org), which has received funding from the European Union's Horizon 2020 research and innovation programme under grant agreement number 633212. This research has been conducted using the UK Biobank Resource under Application Number 19136, and we thank the participants, field workers and data managers for their time and cooperation. ISGlobal is a member of the CERCA Programme, Generalitat de Catalunya. Funding information for this article has been deposited with the Crossref Funder Registry.

\section{References}

1 Sansores RH, Ramírez-Venegas A. COPD in women: susceptibility or vulnerability? Eur Respir J 2016; 47: 19-22.

2 Townsend EA, Miller VM, Prakash YS. Sex differences and sex steroids in lung health and disease. Endocr Rev 2012; 33: 1-47.

3 Dratva J, Gómez Real F, Schindler C, et al. Is age at menopause increasing across Europe? Results on age at menopause and determinants from two population-based studies. Menopause 2009; 16: 385-394.

4 Campbell B, Davis SR, Abramson MJ, et al. Menopause, lung function and obstructive lung disease outcomes: a systematic review. Climacteric 2017; 21: 1-10.

5 Amaral AFS, Strachan DP, Real FG, et al. Lower lung function associates with cessation of menstruation: UK Biobank data. Eur Respir J 2016; 48: 1288-1297.

6 Sapre S, Thakur R. Lifestyle and dietary factors determine age at natural menopause. J Midlife Health 2014; 5: 3-5.

7 Ruth KS, Perry JRB, Henley WE, et al. Events in early life are associated with female reproductive ageing: a UK Biobank study. Sci Rep 2016; 6: 24710. 

London, CRC Press, 2015.

9 Sheehan NA, Didelez V, Burton PR, et al. Mendelian randomisation and causal inference in observational epidemiology. PLoS Med 2008; 5: 1205-1210.

10 Bowden J, Del Greco MF, Minelli C, et al. A framework for the investigation of pleiotropy in two-sample summary data Mendelian randomization. Stat Med 2017; 36: 1783-1802.

11 Sudlow C, Gallacher J, Allen N, et al. UK Biobank: an open access resource for identifying the causes of a wide range of complex diseases of middle and old age. PLoS Med 2015; 12: e1001779.

12 Quanjer PH, Stanojevic S, Cole TJ, et al. Multi-ethnic reference values for spirometry for the 3-95-yr age range: the global lung function 2012 equations. Eur Respir J 2012; 40: 1324-1343.

13 Stolk L, Zhai G, van Meurs JB, et al. Loci at chromosomes 13, 19 and 20 influence age at natural menopause. Nat Genet 2009; 41: 645-647.

14 Stolk L, Perry JR, Chasman DI, et al. Meta-analyses identify 13 loci associated with age at menopause and highlight DNA repair and immune pathways. Nat Genet 2012; 44: 260-268.

15 He C, Kraft P, Chen C, et al. Genome-wide association studies identify loci associated with age at menarche and age at natural menopause. Nat Genet 2009; 41: 724-728.

16 Lunetta KL, D'Agostino RB, Karasik D, et al. Genetic correlates of longevity and selected age-related phenotypes: a genome-wide association study in the Framingham Study. BMC Med Genet 2007; 8: Suppl. 1, S13.

17 Perry JR, Corre T, Esko T, et al. A genome-wide association study of early menopause and the combined impact of identified variants. Hum Mol Genet 2013; 22: 1465-1472.

18 Day FR, Ruth KS, Thompson DJ, et al. Large-scale genomic analyses link reproductive aging to hypothalamic signaling, breast cancer susceptibility and BRCA1-mediated DNA repair. Nat Genet 2015; 47: 1294-1303.

19 Burgess S, Butterworth A, Thompson SG. Mendelian randomization analysis with multiple genetic variants using summarized data. Genet Epidemiol 2013; 37: 658-665.

20 Thompson JR, Minelli C, Del Greco MF. Mendelian randomization using public data from genetic consortia. Int $J$ Biostat 2016; 12: 20150074.

21 Yavorska OO, Burgess S. MendelianRandomization: an R package for performing Mendelian randomization analyses using summarized data. Int J Epidemiol 2017; 46: 1734-1739.

22 Del GMF, Minelli C, Sheehan NA, et al. Detecting pleiotropy in Mendelian randomisation studies with summary data and a continuous outcome. Stat Med 2015; 34: 2926-2940.

23 Bowden J, Davey Smith G, Haycock PC, et al. Consistent estimation in Mendelian randomization with some invalid instruments using a weighted median estimator. Genet Epidemiol 2016; 40: 304-314.

24 Bowden J, Smith GD, Burgess S. Mendelian randomization with invalid instruments: effect estimation and bias detection through Egger regression. Int J Epidemiol 2015; 44: 512-525.

25 Verbanck M, Chen CY, Neale B, et al. Detection of widespread horizontal pleiotropy in causal relationships inferred from Mendelian randomization between complex traits and diseases. Nat Genet 2018; 50: 693-698.

26 Staley JR, Blackshaw J, Kamat MA, et al. PhenoScanner: a database of human genotype-phenotype associations. Bioinformatics 2016; 32: 3207-3209.

27 Palmer TM, Sterne JAC, Harbord RM, et al. Instrumental variable estimation of causal risk ratios and causal odds ratios in Mendelian randomization analyses. Am J Epidemiol 2011; 173: 1392-1403.

28 Amaral AFS, Strachan DP, Burney PGJ, et al. Female smokers are at greater risk of airflow obstruction than male smokers UK Biobank. Am J Respir Crit Care Med 2017; 195: 1226-1235.

29 Tam A, Bates JHT, Churg A, et al. Sex-related differences in pulmonary function following 6 months of cigarette exposure: implications for sexual dimorphism in mild COPD. PLoS One 2016; 11: e0164835.

30 Townsend P, Phillimore P, Beattie A. Health and Deprivation: Inequality and the North. London, Croom Helm, 1988.

31 Real FG, Svanes C, Omenaas ER, et al. Lung function, respiratory symptoms, and the menopausal transition. J Allergy Clin Immunol 2008; 121: 72-80.

32 Jarvis D, Leynaert B. The association of asthma, atopy and lung function with hormone replacement therapy and surgical cessation of menstruation in a population-based sample of English women. Allergy 2008; 63: 95-102.

33 Songür N, Aydin ZD, Oztürk O, et al. Respiratory symptoms, pulmonary function, and reproductive history: Isparta Menopause and Health Study. J Womens Health (Larchmt) 2010; 19: 1145-1154.

34 Hayatbakhsh MR, Najman JM, O'Callaghan MJ, et al. Association between smoking and respiratory function before and after menopause. Lung 2011; 189: 65-71.

35 Triebner K, Matulonga B, Johannessen A, et al. Menopause is associated with accelerated lung function decline. Am J Respir Crit Care Med 2017; 195: 1058-1065.

36 Davies NM, Holmes M V, Davey Smith G. Reading Mendelian randomisation studies: a guide, glossary, and checklist for clinicians. BMJ 2018; 362: k601.

37 Palmer TM, Lawlor DA, Harbord RM, et al. Using multiple genetic variants as instrumental variables for modifiable risk factors. Stat Methods Med Res 2012; 21: 223-242.

38 Brion M-JA, Shakhbazov K, Visscher PM. Calculating statistical power in Mendelian randomization studies. Int J Epidemiol 2013; 42: 1497-1501.

39 Sathish V, Martin YN, Prakash YS. Sex steroid signaling: implications for lung diseases. Pharmacol Ther 2015; 150: 94-108.

40 Pata O, Atis S, Utku Oz A, et al. The effects of hormone replacement therapy type on pulmonary functions in postmenopausal women. Maturitas 2003; 46: 213-218.

41 Hepburn MJ, Dooley DP, Morris MJ. The effects of estrogen replacement therapy on airway function in postmenopausal, asthmatic women. Arch Intern Med 2001; 161: 2717-2720.

42 Cevrioglu AS, Fidan F, Unlu M, et al. The effects of hormone therapy on pulmonary function tests in postmenopausal women. Maturitas 2004; 49: 221-227.

43 Stipic I, Polasek O, Vulic M, et al. Estrogen replacement therapy improves pulmonary function in postmenopausa women with genital prolapse. Rejuvenation Res 2012; 15: 596-600.

44 Mahmoud O, Granell R, Tilling $\mathrm{K}$, et al. Association of height growth in puberty with lung function: a longitudinal study. Am J Respir Crit Care Med 2018; 198 [DOI:10.1164/rccm.201802-0274OC]. 